\title{
Letter on Rapamycin and 3-Methyladenine Influence the Apoptosis, Senescence, and Adipogenesis of Human Adipose- Derived Stem Cells by Promoting and Inhibiting Autophagy: An In Vitro and In Vivo Study
}

\author{
Zuguang Hua ${ }^{1} \cdot$ Peng Wei $^{1}$
}

Received: 8 June 2021 / Accepted: 10 June 2021/Published online: 16 July 2021

(C) Springer Science+Business Media, LLC, part of Springer Nature and International Society of Aesthetic Plastic Surgery 2021

Level of Evidence $V$ This journal requires that authors assign a level of evidence to each article. For a full description of these Evidence-Based Medicine Ratings, please refer to Table of Contents or online Instructions to Authors www.springer.com/00266.

Sir,

We read with great interest the article entitled "Rapamycin and 3-Methyladenine Influence the Apoptosis, Senescence, and Adipogenesis of Human Adipose-Derived Stem Cells by Promoting and Inhibiting Autophagy: An In Vitro and In Vivo Study" by Yang F et al. [1] in Aesthetic Plastic Surgery. In this article, the authors delineated the effect of autophagy on the viability of adipose-derived stem cells (ADSCs) via pretreatment with rapamycin and 3-methyladenine, respectively. The authors' study provided a reference for understanding the role of autophagy in fat grafting.

For the in vitro experiments, the authors treated ADSCs with rapamycin and 3-methyladenine and assessed the autophagy, apoptosis, adipogenesis, proliferation, and senescence. The authors stated that the upregulation of autophagy by rapamycin inhibited the proliferation of ADSCs and its potential effect on fat graft outcome. However, they did not explain why the proliferation of ADSCs declined after a decreased autophagy due to 3-methyladenine pretreatment.

For the in vivo experiments, the authors mixed granular fat with $3.2 * 10^{6}$ cells $/ \mathrm{ml}$ of ADSCs at a volume ratio of 1:19. We would like to know how the concentration and volume ratio of the ADSCs were determined. Besides, the authors injected four groups of mixture into the same mouse, and we feared that the systematic effect caused by the drug absorption would interfere with the discrepancy of different interventions. For the outcome evaluation, we believe that hematoxylin-eosin (HE) staining is basic and helps to evaluate the general structure and quality of the graft.

\section{Declarations}

Conflict of interest The authors declare that they have no conflict of interest to disclose.

Human and Animal Participants This article does not contain any studies with human participants or animals performed by any of the authors.

Informed Consent For this type of study, informed consent is not required.

\section{Reference}

1. Yang F, Du L, Song G et al (2021) Rapamycin and 3-Methyladenine influence the apoptosis, senescence, and adipogenesis of human adipose-derived stem cells by promoting and inhibiting autophagy: an in vitro and in vivo study. Aesthetic Plast Surg 45(3):1294-1309

Publisher's Note Springer Nature remains neutral with regard to jurisdictional claims in published maps and institutional affiliations.

Zuguang Hua

hzg-m2004@126.com

1 Ningbo No1 Hospital, No. 59, Liuting Street, Haishu District, Ningbo 315000, Zhejiang, People's Republic of China 